\title{
ESTUDO DA CINÉTICA DE SECAGEM DAS CASCAS DE BANANAS DAS VARIEDADES NANICA E PRATA
}

\author{
P. O. R. BERTO ${ }^{1}$, J. C. DALCOLLE ${ }^{1}$, L. A. FERREIRA ${ }^{1}$, C. A. CANCIAM ${ }^{2}$, N. C. \\ PEREIRA $^{1}$ e M. F. VIEIRA ${ }^{1}$ \\ ${ }^{1}$ Universidade Estadual de Maringá, Departamento de Engenharia Química \\ 2 Universidade Tecnológica Federal do Paraná - Campus Ponta Grossa, Departamento \\ Acadêmico de Engenharia Química \\ E-mail para contato: pauloodone.rb@gmail.com
}

\begin{abstract}
RESUMO - A cinética de secagem é importante para o dimensionamento e otimização de uma planta industrial de secagem. O objetivo desse trabalho foi estudar a cinética de secagem de cascas de bananas das variedades nanica e prata para posterior uso como bioadsorvente. Foram realizadas secagens a 40, 50, 60, 70 e $80^{\circ} \mathrm{C}$. Observou-se que o tempo necessário para a secagem variou de $90 \mathrm{~min}$, para a secagem a $80^{\circ} \mathrm{C}$, até $330 \mathrm{~min}$, para a secagem a $40^{\circ} \mathrm{C}$. Constatou-se que a variedade da fruta não interferiu na cinética de secagem. Dentre os modelos matemáticos estudados, o modelo Logarítmico foi o que melhor se ajustou aos dados experimentais na maioria das secagens realizadas.
\end{abstract}

\section{INTRODUÇÃO}

A banana é uma das frutas mais consumidas em regiões tropicais e subtropicais (Alkarkhi et al., 2011) e também uma das frutas mais importantes no mundo, levando-se em conta a produção e a comercialização. Para alguns países, corresponde a um produto de exportação responsável por uma parte muito significativa da receita relativa à exportação agrícola (Fioravanço, 2003).

A casca de banana representa de 47 a $50 \%$ do peso total da fruta madura. Segundo Cruz et al. (2009), a casca de banana não apresenta aplicações de ordem industrial, sendo utilizada esporadicamente na alimentação animal.

A casca de banana pode ser processada e convertida em bioadsorvente, apresentando grande potencial de adsorção, pois apresenta elevada área superficial, grande capacidade de intumescimento e excelente resistência mecânica (Annadurai et al., 2002).

Em estudos reportados na literatura, os bioadsorventes, em relação aos adsorventes sintéticos, são de origem natural, e por se tratarem de resíduos agrícolas, não apresentam valor comercial (Vaghetti et al., 2002).

Cascas de banana secas e moídas foram empregadas como bioadsorvente na remoção de íons $\mathrm{Pb}^{+2}, \mathrm{Cd}^{+2}$ e $\mathrm{Cr}^{+4}$ (Anwar et al., 2010; Memon et al., 2009). Também foram utilizadas na remoção de metais pesados, como $\mathrm{Cu}^{+2}, \mathrm{Zn}^{+2}, \mathrm{Co}^{+2} \mathrm{e} \mathrm{Ni}^{+2}$ (Annadurai et al., 2002). 
A secagem de materiais orgânicos consiste na remoção da umidade excessiva contida na estrutura do material por meio da evaporação, comumente utilizando convecção forçada de ar aquecido, visando permitir a manutenção de sua qualidade durante o armazenamento por longos períodos (Almeida et al., 2009). No caso da produção de bioadsorventes, o processo de secagem, além de evitar a degradação do produto, permite que os poros do material estejam livres para que ocorra a adsorção.

Neste sentido, o objetivo desse trabalho foi determinar experimentalmente as curvas de secagem para as cascas de banana das variedades nanica e prata e ajustar diferentes modelos matemáticos aos dados experimentais, em função da temperatura do ar de secagem.

\section{MATERIAL E MÉTODOS}

As bananas foram adquiridas no comércio local. Suas cascas foram cortadas em quadrados de aproximadamente $2 \times 2 \mathrm{~cm}$ e secadas em um módulo experimental disponível no Laboratório de Engenharia Química 2 da Universidade Estadual de Maringá. O módulo consiste em um secador convectivo de fluxo de ar ascendente, aquecido por meio de resistências elétricas, com vazão ajustada. A bandeja é de fundo telado com dimensões 46x46 $\mathrm{cm}$. $\mathrm{O}$ ar aquecido passa pela amostra em sentido perpendicular a mesma, conforme ilustrado na Figura 1.

Figura 1 - Módulo experimental de secagem convectiva

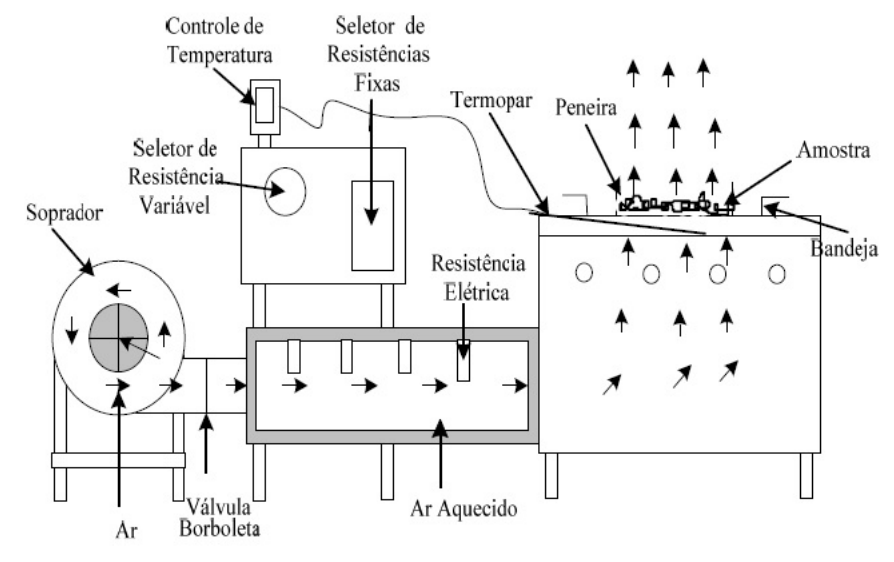

A secagem das cascas foi realizada com velocidade de ar constante de $(1,3 \pm 0,2) \mathrm{m} \cdot \mathrm{s}^{-1} \mathrm{e}$ as temperaturas do ar aquecido foram de $40,50,60,70$ e $80^{\circ} \mathrm{C}$. Para cada uma das temperaturas do ar aquecido foram utilizadas em torno de $200 \mathrm{~g}$ de cascas de bananas nanica e prata. Cada variedade de casca de banana foi colocada em uma peneira laboratorial, Tyler 12, com 20,0 cm de diâmetro. A cada secagem, duas peneiras eram dispostas na bandeja de secagem, uma com as cascas de banana nanica e outra com cascas de banana prata. A área restante na bandeja foi preenchida com papelão de modo que todo ar aquecido passasse pelas amostras a serem secas.

Durante cada secagem, as amostras foram pesadas, em balança analítica com precisão de $0,01 \mathrm{~g}$. A primeira medição da massa de banana nanica foi feita com 2 min de secagem, enquanto que para a casca de banana prata foi com $3 \mathrm{~min}$. Após isso, a cada $2 \mathrm{~min}$ foram 
realizadas as pesagens das massas das cascas durante a secagem até que se atingisse o equilíbrio mássico. Cada pesagem levou em torno de $10 \mathrm{~s}$. Para que o efeito das condições ambientais seja o mesmo durante o processo de secagem, as secagens das cascas de banana nanica e prata foram realizadas simultaneamente.

Para obtenção da massa seca $\left(m_{\sec a}\right)$ empregou-se o método do peso constante em estufa a $105^{\circ} \mathrm{C}$ por um período de 24 horas, em triplicata. A Equação 1 determina a umidade em base seca $(X)$.

$$
X=\left(m_{\text {úmida }}-m_{\sec a}\right) / m_{\sec a}
$$

A Equação 2 indica a umidade adimensional $(Y)$. As curvas de secagem para cada temperatura estudada serão dadas pelo gráfico da umidade adimensional versus o tempo (Coelho e Pinto, 2011).

$$
Y=X(t) / X_{0}
$$

Nesse trabalho foram considerados os modelos de Newton e Lawis (Equação 3), Page (Equação 4), Henderson e Pabis (Equação 5), Logarítmico (Equação 6), Dois Termos (Equação 7) e Henderson e Pabis Modificado (Equação 8). Essas equações foram sugeridas por Ndapeu et al. (2013).

$$
\begin{aligned}
& Y=\exp (-k \cdot t) \\
& Y=\exp \left(-k \cdot t^{n}\right) \\
& Y=a \cdot \exp (-k \cdot t) \\
& Y=a \cdot \exp (-k \cdot t)+b \cdot t \\
& Y=a \cdot \exp \left(-k_{0} \cdot t\right)+b \cdot \exp \left(-k_{1} \cdot t\right) \\
& Y=a \cdot \exp (-k \cdot t)+b \cdot \exp (-g \cdot t)+c \cdot \exp (-h \cdot t)
\end{aligned}
$$

Com base nos dados experimentais e nas Equações 3, 4, 5, 6, 7 e 8 foi utilizado o programa OriginPro 8.0 para determinar os parâmetros dos modelos a partir da análise de regressão não linear. Considerou-se como critério estatístico, o coeficiente de determinação ( $R^{2}$ ), ou seja, quanto mais próximo da unidade for esse coeficiente, melhor é o ajuste do modelo aos dados experimentais. No caso de mais de um modelo apresentar o mesmo valor de coeficiente de determinação, serão calculadas a raiz do erro quadrático médio (RMSE) e a raiz quadrada dos erros (SSE). De maneira que, quanto menor esses parâmetros, melhor é o ajuste do modelo em relação aos dados experimentais. 


\section{RESULTADOS E DISCUSSÃO}

A umidade inicial, em base seca, para as cascas de banana nanica foi de $(9,4448 \pm$ $0,2077) \mathrm{g}$ água/ $\mathrm{g}$ massa seca. Para as cascas de banana prata, a umidade inicial, em base seca, foi de $(7,5087 \pm 0,1596) \mathrm{g}$ água/ $\mathrm{g}$ massa seca.

A Figura 2 ilustra as curvas de secagem para as cascas de banana da variedade nanica, enquanto que a Figura 3, para a variedade prata.

Figura 2 - Curvas de secagem de casca de banana nanica

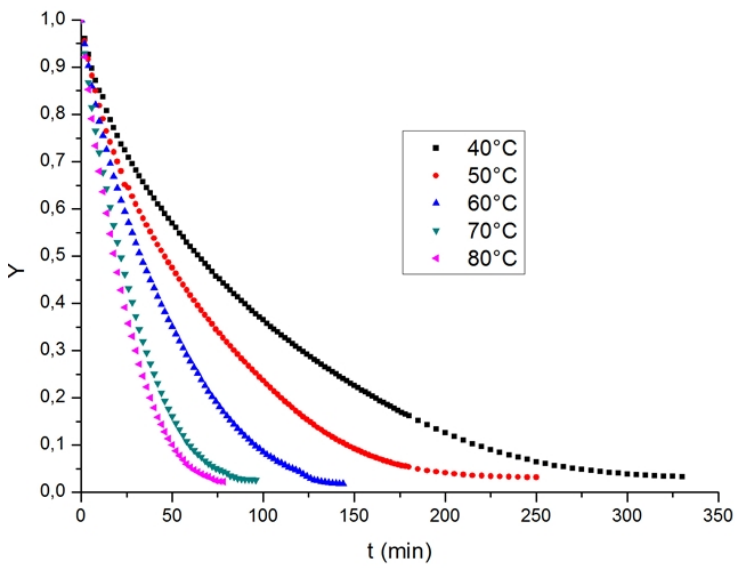

Figura 3- Curvas de secagem de casca de banana prata

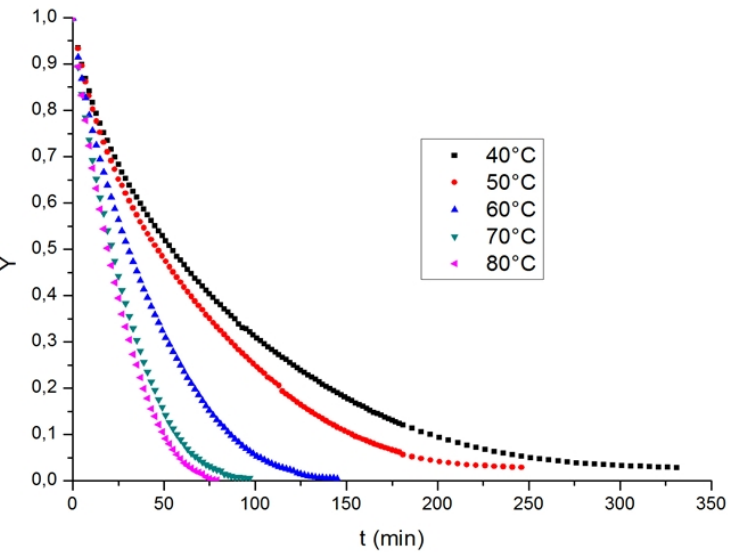

Nessas figuras, observa-se que a cinética de secagem foi fortemente influenciada pela temperatura. Os resultados constatam que a aplicação de uma temperatura mais elevada reduziu significativamente o tempo de secagem. Altas taxas de secagem são alcançadas quando se eleva a temperatura do ar, pois ocorre um aumento no coeficiente de difusão da umidade.

As Tabelas 1 e 2 relacionam, respectivamente, os coeficientes de determinação dos modelos estudados para os dados experimentais de secagem das cascas das variedades nanica e prata.

Tabela 1 - Coeficientes de determinação $\left(R^{2}\right)$ para a variedade nanica

\begin{tabular}{cccccc}
\hline Modelo & $40^{\circ} \mathrm{C}$ & $50{ }^{\circ} \mathrm{C}$ & $60^{\circ} \mathrm{C}$ & $70^{\circ} \mathrm{C}$ & $80^{\circ} \mathrm{C}$ \\
\hline Newton e Lawis & 0,9915 & 0,9952 & 0,9951 & 0,9948 & 0,9941 \\
Page & 0,9961 & 0,9960 & 0,9963 & 0,9983 & 0,9983 \\
Henderson e Pabis & 0,9974 & 0,9970 & 0,9951 & 0,9955 & 0,9950 \\
Logarítmico & 0,9977 & 0,9976 & 0,9992 & 0,9982 & 0,9986 \\
Dois Termos & 0,9973 & 0,9969 & 0,9950 & 0,9953 & 0,9947 \\
Henderson e Pabis & 0,9973 & 0,9969 & 0,9948 & 0,9951 & 0,9944 \\
Modificado & & & & &
\end{tabular}

Observa-se na Tabela 1 que o modelo Logarítmico apresentou o maior valor para o coeficiente de determinação nas temperaturas de secagem de $40,50,60$ e $80^{\circ} \mathrm{C}$; indicando que 
para essas temperaturas de secagem, o modelo Logarítmico ajustou-se melhor aos dados experimentais. Na temperatura de secagem de $70^{\circ} \mathrm{C}$, o modelo de Page apresentou o maior valor para o coeficiente de determinação.

Tabela 2 - Coeficientes de determinação $\left(R^{2}\right)$ para a variedade prata

\begin{tabular}{cccccc}
\hline Modelo & $40^{\circ} \mathrm{C}$ & $50^{\circ} \mathrm{C}$ & $60^{\circ} \mathrm{C}$ & $70^{\circ} \mathrm{C}$ & $80^{\circ} \mathrm{C}$ \\
\hline Newton e Lawis & 0,9903 & 0,9939 & 0,9926 & 0,9900 & 0,9863 \\
Page & 0,9977 & 0,9957 & 0,9951 & 0,9959 & 0,9962 \\
Henderson e Pabis & 0,9980 & 0,9970 & 0,9927 & 0,9911 & 0,9888 \\
Logarítmico & 0,9980 & 0,9978 & 0,9986 & 0,9978 & 0,9983 \\
Dois Termos & 0,9979 & 0,9970 & 0,9925 & 0,9907 & 0,9882 \\
Henderson e Pabis & 0,9979 & 0,9969 & 0,9922 & 0,9903 & 0,9875 \\
Modificado & & & & & \\
\hline
\end{tabular}

$\mathrm{Na}$ Tabela 2, o modelo Logarítmico apresentou o maior valor para o coeficiente de determinação nas temperaturas de secagem de $50,60,70$ e $80^{\circ} \mathrm{C}$; indicando que para essas temperaturas de secagem, o modelo Logarítmico ajustou-se melhor aos dados experimentais. A $40^{\circ} \mathrm{C}$, os modelos de Henderson e Pabis e Logarítmico obtiveram o mesmo valor para o coeficiente de determinação. Para o modelo de Henderson e Pabis, a raiz do erro quadrático médio (RMSE) foi igual a $1,0982 \times 10^{-2}$ e a raiz quadrada dos erros (SSE) foi de $1,2260 \times 10^{-4}$. Para o modelo Logarítmico, a raiz do erro quadrático médio foi de $1,0982 \times 10^{-2}$ e a raiz quadrada dos erros equivalente a $1,2360 \times 10^{-4}$. Dessa forma, os resultados indicam que para a temperatura de $40^{\circ} \mathrm{C}$, o modelo de Henderson e Pabis ajusta-se melhor aos dados experimentais.

Aplicando um teste $\mathrm{t}$ para comparação dos valores do coeficiente de determinação constatou-se que nas Tabelas 1 e 2 os valores do coeficiente de determinação não apresentam diferença significativa ao nível de 5\% de significância.

A Tabela 3 relaciona as constantes encontradas pela análise de regressão não linear para os modelos matemáticos que melhor se ajustaram aos dados experimentais.

Tabelas 3 - Constantes dos melhores modelos ajustados aos dados experimentais

\begin{tabular}{cccccc}
\hline Variedade $($ Temperatura $)$ & $\mathrm{a}$ & $\mathrm{b}$ & $\mathrm{k}$ & $\mathrm{n}$ & Modelo \\
\hline Nanica $\left(40^{\circ} \mathrm{C}\right)$ & 0,93213 & $-5,49742 \times 10^{-5}$ & 0,00941 & --- & Logarítmico \\
Prata $\left(40^{\circ} \mathrm{C}\right)$ & 0,92568 & --- & 0,01113 & --- & Henderson e Pabis \\
Nanica $\left(50^{\circ} \mathrm{C}\right)$ & 0,95286 & $-8,2647 \times 10^{-5}$ & 0,01396 & --- & Logarítmico \\
Prata $\left(50^{\circ} \mathrm{C}\right)$ & 0,94004 & $-9,98725 \times 10^{-5}$ & 0,01328 & --- & Logarítmico \\
Nanica $\left(60^{\circ} \mathrm{C}\right)$ & 0,97982 & $-3,69131 \times 10^{-4}$ & 0,02006 & --- & Logarítmico \\
Prata $\left(60^{\circ} \mathrm{C}\right)$ & 0,98285 & $-3,97468 \times 10^{-4}$ & 0,02196 & --- & Logarítmico \\
Nanica $\left(70^{\circ} \mathrm{C}\right)$ & --- & --- & 0,02361 & 1,10909 & Page \\
Prata $\left(70^{\circ} \mathrm{C}\right)$ & 1,00352 & $-6,38627 \times 10^{-4}$ & 0,03277 & --- & Logarítmico \\
Nanica $\left(80^{\circ} \mathrm{C}\right)$ & 1,00826 & $-6,20886 \times 10^{-4}$ & 0,03872 & --- & Logarítmico \\
Prata $\left(80^{\circ} \mathrm{C}\right)$ & 1,01313 & $-1,03 \times 10^{-3}$ & 0,03614 & --- & Logarítmico \\
\hline
\end{tabular}




\section{CONCLUSÃO}

A cinética de secagem das cascas de bananas das variedades nanica e prata são fortemente influenciadas pela temperatura do ar aquecido. Os resultados indicam que a aplicação de uma temperatura mais elevada reduziu significativamente o tempo de secagem.

Observou-se que o tempo necessário para a secagem das cascas das variedades nanica e prata variou de $90 \mathrm{~min}$ (para o ar aquecido a $80^{\circ} \mathrm{C}$ ) a $330 \mathrm{~min}$ (para o ar aquecido a $40^{\circ} \mathrm{C}$ ).

O modelo Logarítmico foi o que melhor se ajustou aos dados experimentais na maioria das secagens realizadas.

\section{REFERÊNCIAS}

ALKARKHI, A. F. M.; RAMLI, S.; YONG, Y. S.; EASA, A. M.; Comparing physicochemical properties of banana pulp and peel flours prepared from green and ripe fruits. Food Chemistry, v. 129, p. 312-318, 2011.

ALMEIDA, D. P.; RESENDE, O.; COSTA, L. M.; MENDES, U. C.; SALES, J. F. Cinética de secagem do feijão adzuki. Global Science and Technology, v. 2, p. 72-83, 2009.

ANNADURAI, G.; JUANG, R. S.; LEE, D. J.; Adsorption of heavy metals from water using banana and orange peels. Water Science and Technology, v. 47, p. 185-190, 2002.

ANWAR, J.; SHAFIQUE, U.; ZAMAN, W. U., SALMAN, M.; DAR, A.; ANWAR, S. Removal of $\mathrm{Pb}(\mathrm{II})$ and $\mathrm{Cd}(\mathrm{II})$ from water by adsorption on peels of banana. Bioresource Technology, v. 101, p. 1752-1755, 2010.

COELHO, K. D.; PINTO, L. A. A. Caracterização da secagem convectiva de tomate (Lycopersicon esculentum Mill) em camada delgada. Vetor, v. 21, p. 5-21, 2011.

CRUZ, M. A. R. F. da Utilização da casca de banana como biossorvente. 67p., Dissertação (Mestrado em Química), Universidade Estadual de Londrina, Londrina, 2009.

FIORAVANÇO, J. C. Mercado mundial de banana: produção, comércio e participação brasileira. Informações Econômicas, v. 33, n. 10, 2003.

MEMON, J.R.; MEMON, S.Q. BHANGER, M.I.; EL-TURKI, A.; HALLAM, K.R.; ALLEN, G.C. Banana peel: A green and economical sorbent for the selective removal of $\mathrm{Cr}$ (VI) from industrial wastewater. Colloids and Surfaces B: Biointerfaces, v. 70, p. 232-237, 2009.

NDAPEU, D.; NJEUGNA, E.; BISTAC, S. B.; DREAN, J. Y.; FOGUE, M.; FOBA, J. N. Experimental study of the drying kinetics of the coconut of Cameroon. Materials Sciences and Applications, v. 4, p. 822-830, 2013.

VAGHETTI, J. C. P. Utilização de biossorventes para remediação de efluentes aquosos contaminados com íons metálicos. 99p., Tese (Doutorado em Química), Universidade Federal do Rio Grande do Sul, Porto Alegre, 2009. 